\title{
Science and technology excimer laser complex and examples of its applications
}

\author{
S.M.Baschenko*, I.V.Blons'kiy. \\ Institute of Physics of NAS of Ukraine, 46, prospect Nauki, 03039, Kyiv, Ukraine. \\ *-E-mail: baschenk@iop.kiev.ua
}

\begin{abstract}
Science and technology multi-purpose, wide-functional excimer laser complex consisting of two precisely and fine synchronized excimer lasers and assembled on the common optical table and added by high efficient Stimulated Raman Scattering converter and both working by a scheme "oscillator - amplifier" is created. Due to these the Complex is able to generate highest quality radiation simultaneously at a few wavelengths with its definite intensities ratio.
\end{abstract}

Radiation of this complex has next parameters: main wavelengths of radiation $-193,222$, 248,308 and $351 \mathrm{~nm}$; radiation pulse duration in the range of $20 \ldots 30 \mathrm{~ns}$; pulse energy at the main wavelengths $-20-30 \mathrm{~mJ}$; bandwidth of radiation line is less than $0.1 \mathrm{~cm}^{-1}$; divergence of radiation is less than $10^{-4}$ radians; fine and smooth tuning within the part of the respective excimer molecule luminescence band - 0.5-4.0 nm; discrete SRS-conversion (tens lines) within the region from 180 to $800 \mathrm{~nm}$; efficiency of SRS-conversion in defined SRScomponent - up to $30-50 \%$; pulse energy at the additional wavelength in dependence on number of emitted components is about $1-10 \mathrm{~mJ}$; repetition rate - up to $50 \mathrm{pps}$.

Highest quality of complex's radiation (spectral brightness up to $10^{12} \mathrm{~W} / \mathrm{nm} \cdot$ steradian) provides its widest application prospects and allows one to achieve power density up to $10^{13-14} \mathrm{~W} / \mathrm{cm}^{2}$ in a focal spot. Three examples of complex application, namely: for remote sensing of atmosphere, high-precision processing of super-hard non-metallic materials and for porous-silicon luminescence properties modification are reported.

Keywords: excimer laser, Stimulated Raman Scattering conversion, atmosphere sensing, sapphire high-precision processing, porous silicon luminescence.

Paper received 11.01.00; revised manuscript recieved 12.01.00; accepted for publication 12.01.00.

\section{Introduction}

For modern investigation in laser both science and technology areas it is desirable to operate with if not universal but at least multi-purposes, multi-functional device: with ability to generate power, narrow-line, sharply directed radiation and desirably simultaneously at a few wavelengths, to convert or vary generated wavelengths in wide spectral region, to tune finely and smoothly generated wavelengths, to vary its intensities, and so on.

We hope to add similar device into the researcher's arsenal.

One of the most perspective laser sources out-perform other ones under definite conditions is the excimer lasers and complexes on its base. Such a situation occurs due to a few circumstances: constants of interaction of light with a matter increases in some power with wavelength de- creasing; area of interaction determines by transversal dimension of focused beam, which is principally limited by and proportional to wavelength; it is excimer lasers that are now the most powerful UV-radiation sources meeting and providing majority of these conditions.

\section{Experimental part}

\subsection{Complex construction}

A single excimer laser with a simple plane-parallel resonator has output radiation of bad quality. Implementation of unstable telescopic resonator provides output radiation with low divergence that is close to diffraction limit. But narrow bandwidth radiation may be obtained in a single excimer laser only at the cost of considerable decreasing of output energy. 


\section{S.M.Baschenko, I.V.Blons'kiy: Science and technology excimer laser complex and...}

Therefore, on common optical table we have joined two specially designed excimer lasers into united complex. It consists of two synchronized excimer lasers assembled and working by using the well-known «oscillator-amplifier» scheme (Fig. 1). It is necessary to note that for effective combining these two lasers in one complex their special development was carried out. The essential point of it consists in vertical configuration of a laser head contrary to the standard horizontal one, and purpose is in achievement of minimal width of a laser head. As a result of this development it became possible to group the laser block in width only $240 \mathrm{~mm}$ (!). This fact has allowed us to place all optical elements of the «oscillator-amplifier» optical scheme on a common, established on a few invar cores, optical plate of width only $600 \mathrm{~mm}$ (Photo 1). This way enabled the achievement of effective optical joining of lasers in the complex with high stability of radiation parameters. Such an effective optical joining cannot be achieved with simple association of two lasers with a typical width $800 \mathrm{~mm}$; you can see in this case that an optical plate would have a width more than $1600 \mathrm{~mm}$ with all following deficiencies: vibrations, weight, etc.

Due to these elaboration, radiation of the excimer laser complex has excellent energetic (20-30 mJ), spatial (low divergence reaching $Q \sim 10^{-4 \ldots 5}$ radians), temporary $(t \approx 25$ nanosec $)$ and spectral characteristics $\left(\Delta \lambda \leq 0.1 \mathrm{~cm}^{-1}\right)$. It is worth to note, that the "oscillatoramplifier» scheme can be easily transformed into the simplified scheme «oscillator» with the use of the unstable telescopic resonator by simple replacement of only one optical element. Such a simplified excimer irradiator scheme can be sufficient in the case, when necessity for

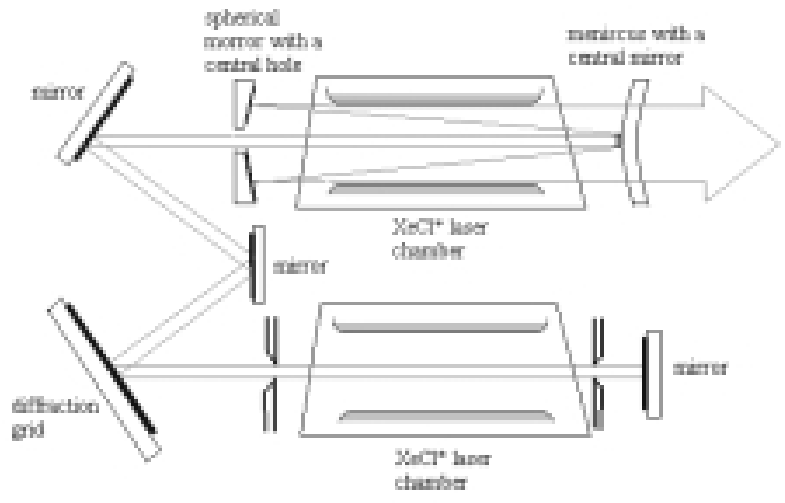

Fig. 1. Principal scheme of most widely used optical scheme «master oscillator - power amplifier», consisting of two precisely and rigidly synchronized excimer lasers, one of which generates the highest quality radiation and the second one amplifies it.

the fine and smooth tuning of the emitting lines will be out of interest.

The excimer laser complex is prepared to work with chlorine and fluorine mixtures and therefore is able to generate radiation of several basic wavelengths -193 , 222, 248, 308 and $351 \mathrm{~nm}$. If not mentioned especially, all preliminary experiments was performed with $\mathrm{XeCl}^{*}$ active gas media. When other media would be used the results will be similar in principle.

In this case, radiation with wavelength $308 \mathrm{~nm}$ will be generated. In the case of one laser with the plane-parallel or unstable telescopic resonator it usually will simultaneously generate four vibration lines $0-0,0-1,0-2,0-3$ with wavelengths $307.7 ; 307.96 ; 308.21$ and $308.46 \mathrm{~nm}$,

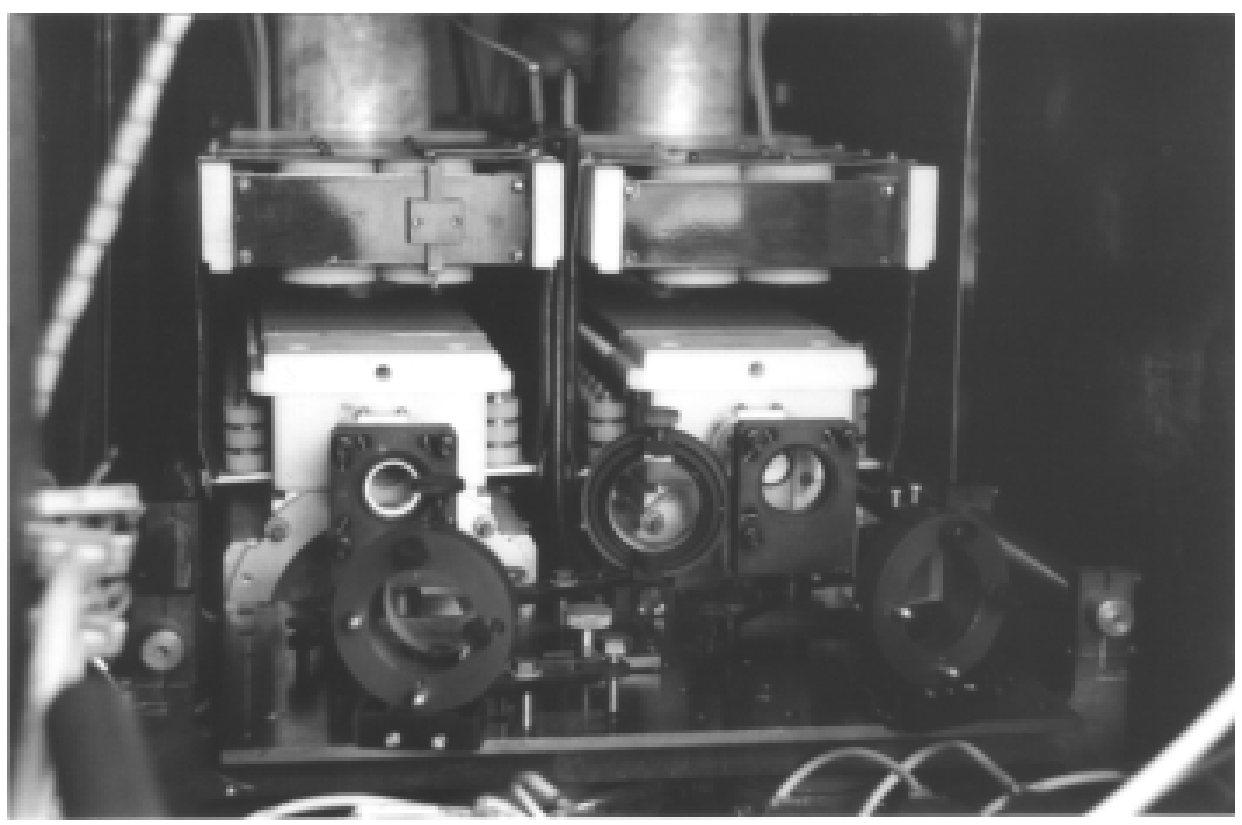

Photo 1. Back view of excimer laser complex - five optical elements of «master oscillator - power amplifier» scheme is mounted on common optical table just of $500 \mathrm{~mm}$ width: diaphragm, mirrors, diffraction grid. 


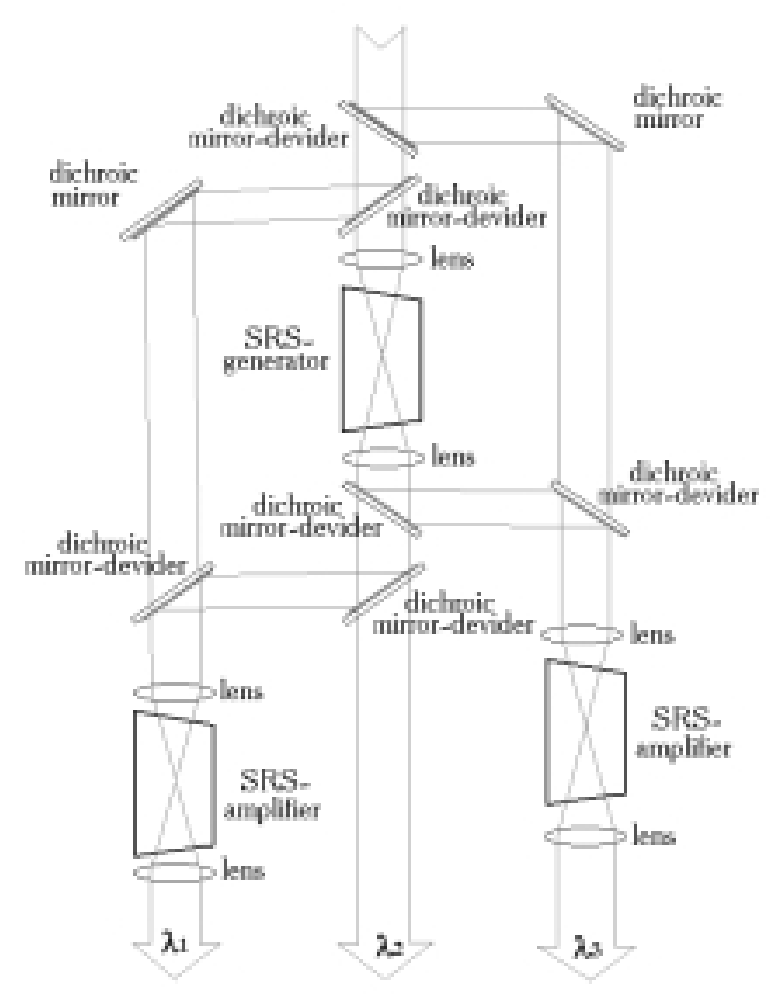

Fig. 2. Optical scheme of SRS-converter assembled by scheme «oscillator-amplifier»: «Oscillator» generates maximal number of SRS-components, a few allocated of which should be amplified in the «Amplifier».

respectively, with intensities which are distributed approximately as 1:5:4:1 [1]. In the case of the complex implication it will generate one supernarrow line with spectral width about $0.1 \mathrm{~cm}^{-1}$ with an opportunity of its fine and smooth tuning within the limits of a part of $\mathrm{XeCl}^{*}$ molecule's luminescence band $\sim 307.4-308.6 \mathrm{~nm}$.

Last opportunity may be very important, necessary in many spectroscopic applications. This opportunity allows to detect distantly the molecular pollutants or small atmosphere components. The typical absorption spectrum of majority of them [2] in its bands' wings often has a vibration al (or of other nature) structure, that requires the thin and smooth tuning of a probing radiation wavelength.

To emit additional lines, the conversion based on Stimulated Raman Scattering(SRS) phenomena will be used. It is also necessary to note, that we have a possibility to realize it both in the simplified variant of «oscillator» and in that of «oscillator-amplifier» (Fig. 2). In the first case it is possible to receive, basically, two first Stokes' and anti-Stokes' SRS-components without an opportunity to vary their intensities - the ratio will be determined by an appropriate levels population. The variant «oscillator-amplifier» is much more complex and difficult in realization, but only it provides an opportunity of high effective SRS-conversion in the certain higher SRScomponent and an essential variation of its intensities.
We intend to choose the «oscillator-amplifier» scheme, which, as it follows from its title, includes the circuit «generator».

The SRS-conversion will be carried out as follows. The beam emitted from an excimer laser or the complex will be shared on a few beams with a certain ratio of intensities. One of them will be directed in a gas tube of the SRS-oscillator, where under the focusing in compressed hydrogen $\mathrm{H}_{2}$ (or other SRS-active medium) will be used for generation of SRS-components with the greatest possible number. At this gas tube the conditions (pressure, focusing angle etc.), optimized especially for generation of the greatest possible SRS-component number will be provided. Of all the SRS-components, which will be generated, the necessary SRS-components will be allocated using appropriate selective optical elements. These selected «seed» SRS-components will be directed into appropriate gas tubes of the SRS-amplifiers, where simultaneously other pumping $(308 \mathrm{~nm})$ beam will come too. A significant amplification of the seed SRS-component will occur and the conditions optimum for the process of amplification will be supported here. After exit from the SRS-converter beams will be spectrally filtered and spatially corrected.

The preliminary experiments have demonstrated as an opportunity of achievement of high - up to $50 \%-$ quantum efficiencies of the SRS-conversion in the certain SRS-component as the peculiarities of «oscillator-amplifier» scheme realization.

Offered scheme of the multi-purposes, wide-functional, multi-frequency laser complex construction on a base of the excimer lasers complemented by SRS-converters, in our opinion, has certain advantages over a widely practised schemes with use of solid-state converters, namely:

- strong receptor-membrane binding, preferably via covalent coupling;

- the opportunity to emit simultaneously not only several lines but an opportunity to realize practically any ratio between their intensities can be provided;

- there is an opportunity of simultaneous both broadband (tens and hundreds nanometers) discrete transformation and the fine, smooth tuning of all emitted lines within the part of an excimer molecule luminescence band. The latter can be important in majority of spectroscopic applications: for instance, in the case of the presence of a characteristic «structure» in the wings of absorption bands (see above);

- there is an opportunity of effective transformation in the short-wave region of the spectrum, where main absorption of molecular pollutants and small atmosphere constituents occur. Besides, the work in the UV region of a spectrum gives us a basic opportunity to carry out the day-time measurements, too;

- in comparison with a solid-state converters the gas SRS-converters have much higher thresholds of optical damage and do not fail with their casual excess, moreover these also have much wider and less selective spectral ranges of work and do not require any matching with a source of pump radiation; 


\section{S.M.Baschenko, I.V.Blons'kiy: Science and technology excimer laser complex and...}

- the efficiency of SRS-transformation in a general case is proportional to $\lambda^{-4}$, which also creates advantages when working in UV-region of a spectrum;

Designed complex may be successfully implemented in various area: mainly into investigations of radiationmatter interactions under the highest power density - up to $10^{13-14} \mathrm{~W} / \mathrm{cm}^{2}$; in remote sensing (distance gas analyzing), since it has power enough for probing over any reasonable distances; in precision non-contact processing of a super-hard, high-melting and transparent in wide spectral range materials; in various materials treatment and its properties modification, and so on.

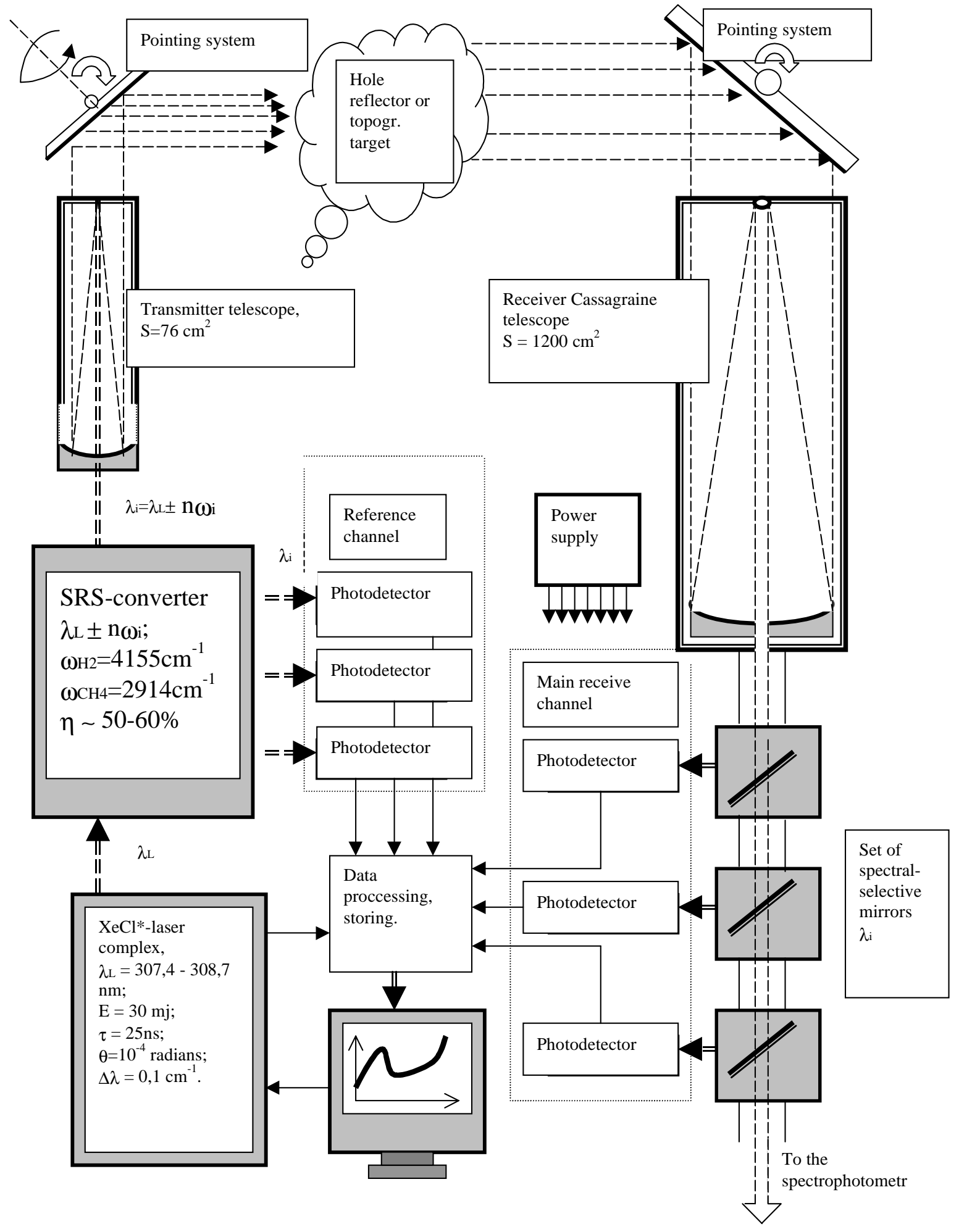

Fig. 3. Optical scheme of multi-functional, multi-purposes Lidar equipped with multi-frequency transmitter. Transmitter consists of Excimer laser Complex added by SRS-converter and both working by «oscillator-amplifier» scheme. 


\subsection{Examples of Complex application}

\subsubsection{Atmosphere remote sensing}

The physical basis of any subjects remote sensing is processes of interaction of substance with radiation (light) [3]: scattering (Rayleigh's, Raman's, Mie's, resonance), fluorescence, absorption or even simple reflection. Using some of these processes, the plenty of various methods is developed and implemented: absorption, differential absorption, differential absorption with a scattering, fluorescence, Raman etc. It is clear that radiation (light) source primarily determines main features, characteristics and thus possibilities of remote sensing apparatus. Receiving part of apparatus only realizes possibilities laid in apparatus groundwork by radiation source. It is well-known that small atmosphere constituents, pollutants, aerosols have a complex absorption spectra with band, strip and line structure. Therefore, LIDAR transmitter must provide conversion and tuning of generated wavelengths.

Nowadays, the most informative and most universal is the method of differential absorption with a scattering. It is a logic development of a differential absorption method and it differs from the first ones, as far as the back-scattered (and also weaken by absorption) signal is received here. It is well-known that while propagating in atmosphere radiation undergoes both an absorption and
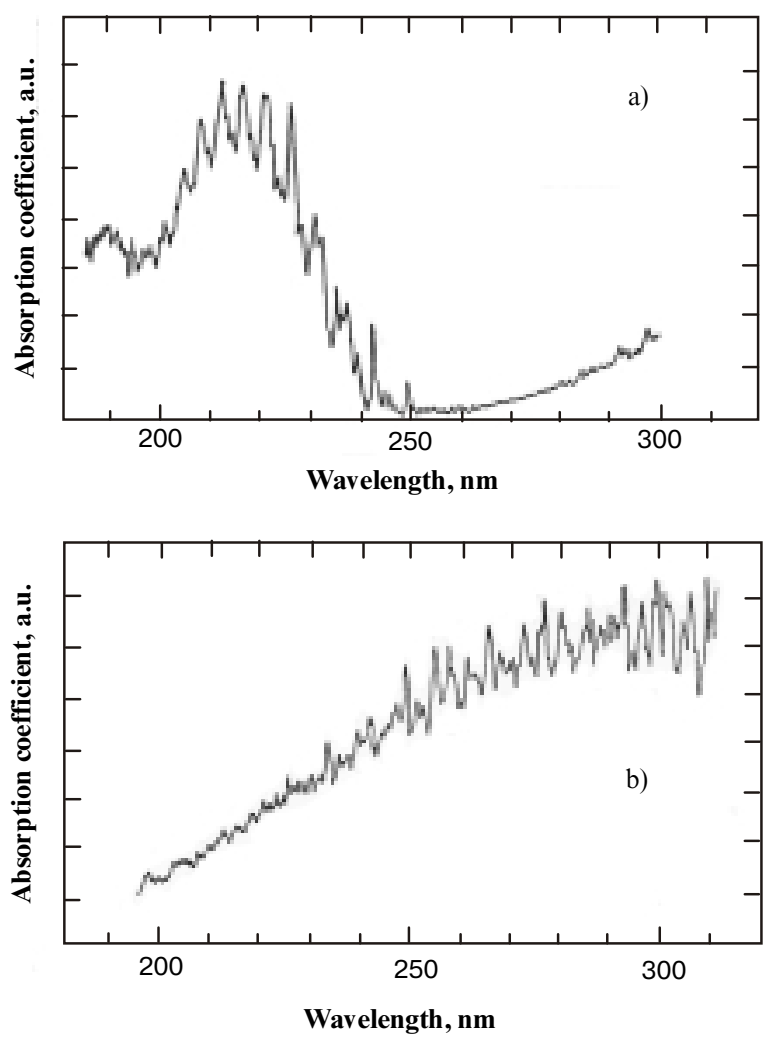

Fig. 4. Absorption coefficient of both sulfur (a) and nitrogen (b) dioxide $v s$ wavelength, measured at $25^{\circ} \mathrm{C}$. scattering (including back) by the molecules and aerosol particles of the atmosphere. It is obvious, that in case of acceptance of the back-scattered signal, the temporary and peak ratios of the receipted signals themselves carry important information on both absorbing and scattering components. The latter circumstance also enables extraction of information about spatial distribution of absorbing pollutants and/or small atmosphere constituents. This opportunity can be realized in the case of probing by short pulses of radiation - you see spatial resolution there cannot be less than the length of a radiation pulse - and at least at two proper wavelengths. The more these lines will be, the wider covered spectral region, the wider conversion and tuning ability, the better: all of these provide possibility of simultaneous measuring several atmosphere components or pollutants, thus enabling correlation and/or transformation process to be recognized.

Proposed laser complex can serve as a three-frequency (potentially - multifrequency) power irradiating system for LIDAR. Block-scheme of such an application is represented in Fig. 3: irradiating subsystem will generate three beams with wavelengths 273,308 and $353 \mathrm{~nm}$ and necessary power parameters $E_{1} \approx E_{2} \approx E_{3} \approx 1-5$ millijoules and other necessary spectral, temporal and spatial parameter. Coming out from an irradiating subsystem these beams are joined or close and directed to a telescopecollimator reducing the divergence of probing beams. From a telescope-collimator the beams are directed to the pointing system, a main element of which is a rotary mirror with two angles of turn - on the height and on the azimuth.

We plan to start from the monitoring of two most important pollutants in the urban air, nitrogen oxides $\mathrm{NO}_{2}$ and sulfur oxides $\mathrm{SO}_{2}$. UV absorption spectra of these gases are studied and known well: they consist of wide band with separate vibrational-rotational lines of a weak structure Fig. 4 [4]. This allows to choose two closely situated wavelengths with considerably differed absorption cross-sections.

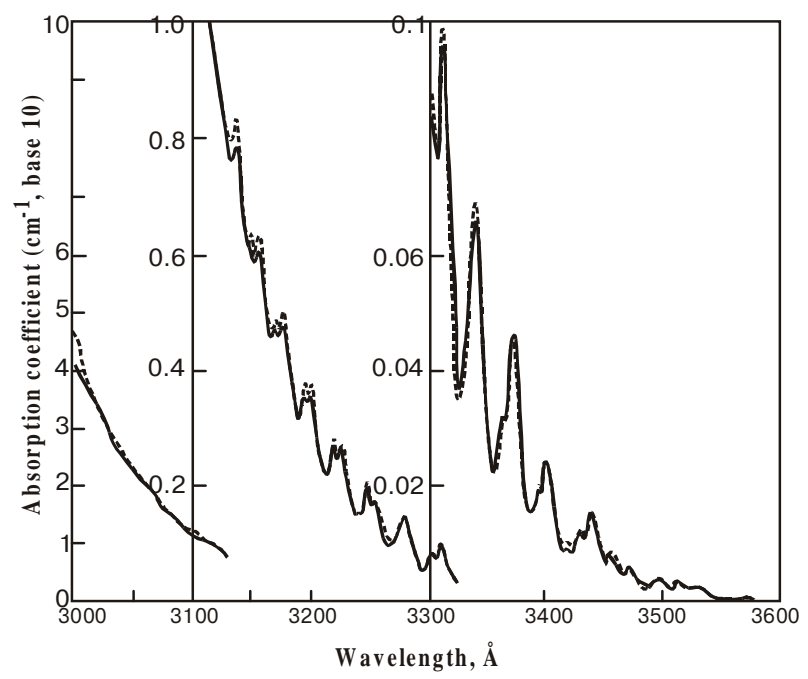

Fig. 5. Fragment of ozone absorption coefficients in spectral region 3000-3600A (the so-called Hartley-Huggins bands).

SQO, 3(1), 2000 


\section{S.M.Baschenko, I.V.Blons'kiy: Science and technology excimer laser complex and...}

The first step will be an attempt to measure $\mathrm{SO}_{2}$ content directly by the DA method using two most intensive lines of the $\mathrm{XeCl}$ laser: $\lambda_{1}=307.96$ and $\lambda_{2}=308.21 \mathrm{~nm}$. Absorption of $\mathrm{SO}_{2}$ interferes with $\mathrm{O}_{3}$, but dispersion of the cross-sections of $\mathrm{O}_{3}$ in the range of $307-309 \mathrm{~nm}$ is by 2 orders of magnitude less and practically negligible. Calculations confirmed the possibility of $\mathrm{SO}_{2}$ trace detection on the distance of $2-3 \mathrm{~km}$ at the level of $10^{-8}$. This is approximately ten-fold comparing to its natural concentration that satisfies demands of ecological control.

The second step will be an attempt to measure $\mathrm{NO}_{2}$ and $\mathrm{SO}_{2}$ content simultaneously by the DA method using the $\mathrm{XeCl}$ laser radiation of $308 \mathrm{~nm}$, supplemented with the SRS components: $1 \mathrm{~S}-353 \mathrm{~nm}$ and $1 \mathrm{AS}-273 \mathrm{~nm}$. Absorption of $\mathrm{NO}_{2}\left(\mathrm{~A}^{2} \mathrm{~B}_{2}-\mathrm{X}^{2} \mathrm{~A}_{1}\right.$ transitions $)$ has no competitors in the blue range of wavelengths. $\mathrm{SO}_{2}$ has a wide band of absorption $\left(\mathrm{A}^{1} \mathrm{~B}_{1}-\mathrm{X}^{1} \mathrm{~A}_{1}\right.$ transitions) which involves the $1 \mathrm{AS}-273 \mathrm{~nm}$ component with the absorption crosssection of $\sigma=8 \cdot 10^{-19} \mathrm{~cm}^{2}$. Near the line of $307.5 \mathrm{~nm}$ the $\mathrm{SO}_{2}$ absorption cross-section decreases to $\sigma=2 \cdot 10^{-19} \mathrm{~cm}^{2}$. At the same time, $\mathrm{NO}_{2}$, on the contrary, has the absorption cross-section $\sigma=1.7 \cdot 10^{-19} \mathrm{~cm}^{2}$ near the line of 308 $\mathrm{nm}$, and $\sigma=5 \cdot 10^{-19} \mathrm{~cm}^{2}$ - for the $1 \mathrm{~S}-353 \mathrm{~nm}$ component. Such large difference in absorptions of $\mathrm{NO}_{2}[\sigma(353) /$ $\sigma(308)]$ and $\mathrm{SO}_{2}[\sigma(273) / \sigma(308)]$ on the respective wavelengths allow to have a high sensitivity of this LIDAR.

In the receiving optical system at the first stages a Newton-type telescope-reflector with a main mirror with a diameter of $380 \mathrm{~mm}$ will be implicated. The optical signal, accepted by it, then will spectrally share on the monochromatic beams with various wavelengths, each of them will be directed on the appropriate photodetectors. The signals from photodetectors will be processed in the computerized analytical system accordingly to a chosen method.

Besides, the offered complex will permit, in the nearest future, to proceed to attempts of carrying out the ozone and aerosols probing. It is this spectral range (273-308$352 \mathrm{~nm}$ ) where ozone has quickly decreasing absorption values. This absorption belongs to the so-called Hartley and Huggins bands, the general view of which is given in Fig. 5 [5].

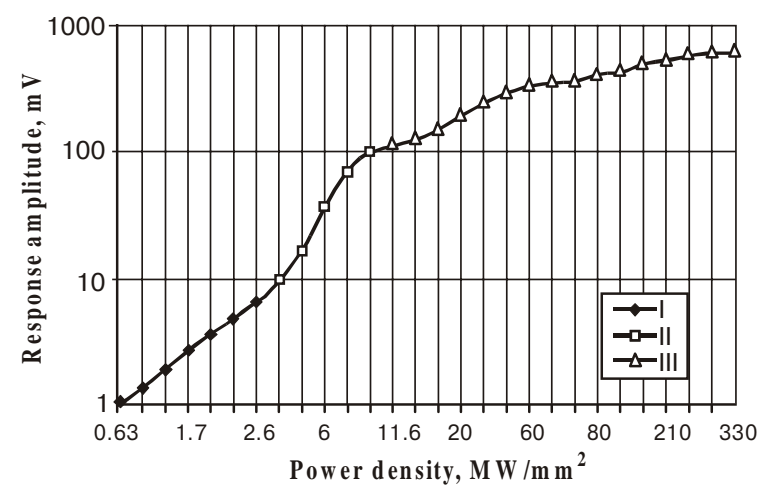

Fig. 6. Typical dependence of the pulsed photo-acoustic response amplitude $\mathrm{U}_{\mathrm{ap}}$ on power density $\mathrm{W}$ of laser radiation for sapphire substrate sample.

\subsubsection{Procession of sapphire}

Involvement of super-hard, high-melting non-metallic materials (such a sapphire, ceramic, silica etc.) into a wide range of applications (electronics, medical apparata, etc.) makes the task of precise processing of such materials to be of high practical importance. For this purpose it seems to be promising to make use of laser radiation under the highest power density and in non-contact and nonthermal regimes of interaction, namely, in the so-called «photoablation» regime. In recent work [6] and in the present work possibilities and advantages of such an approach are demonstrated when the eximer $\mathrm{XeCl}^{*}$ laser complex has been employed as a source of radiation. It should be noted that the laser radiation wavelength is a critical parameter determining the accuracy limit of processing. Using methods of photoacoustical analysis among other ones allows to determine the different regimes of radiation-material interaction with high accuracy.

The studied sapphire samples were of $15 \times 10 \times 1 \mathrm{~mm}^{3}$ size. These have been attached to $X-Y$ adjustable mounts situated in special evacuated chambers fitted with portholes. This enables to move precisely the specimen along and across the light beam with a measurable shift of 0.01 $\mathrm{mm}$.

To detect the acoustic response to the light irradiation the piezotransducer was attached to a back of the sapphire plate. The latter was simultaneously used as a soundguide. Registration of the signal's shape was performed by means of the digital oscilloscope (bandwidth $100 \mathrm{MHz}$ ). Special system for shielding from an external induction noise was employed. The minimum detectable signal level was about $1 \mathrm{mV}$ with the external induction noise of $0.3 \mathrm{mV}$. This is the signal-to-noise ratio that determines the lower limit of the light power density employed $-0.6 \mathrm{MW} / \mathrm{mm}^{2}$.

One of the important aspects of laser processing is determination of the threshold of failure of the processed material. This task is also of importance out of frame of the problem considered in this work if one considers the general problem of interaction of laser radiation with a matter. There exists a number of methods for the failure threshold determination. In the present work the possibilities of the method are demonstrated using the PA effect - generation of elastic waves in a medium affected by irradiation of light of variable intensity. As shown by means of detection of sound waves generated under the substance irradiation with nanosecond laser pulses and analysing the dependence of amplitude of sound wave versus power density of radiation, it becomes possible not only to detect the substance's failure threshold (which preceeds the visual observation of the failure), but also to track a change of sound generation mechanisms.

In Fig. 6 a dependence of the PA response amplitude on the excitation power density $W$ is adduced. As seen, this dependence is of essentially non-linear shape. It may be conventionally devided by three characteristic sections at this curve (I, II, and III). 


\section{S.M.Baschenko, I.V.Blons'kiy: Science and technology excimer laser complex and...}

Within the section I a linear connection between the values of $U_{p a}$ and $\mathrm{W}$ is conserved. With taking into account wavelength and width of the laser pulse it may be supposed that within the limits of this section of excitation densities, two mechanisms of different partial contribution may be responsible for the PA signal formation: the thermoelastic and strictional ones. Any damages of the sapphire plate are not observed in this region (I).

A visual evidence of the sample's damage was observed under power density which corresponds approximately to the middle point of the section II $\left(W=6-8 \mathrm{MW} / \mathrm{mm}^{2}\right)$, i.e., in the region of superlinear dependence of $U_{p a}$ on $W$. This change of nature of the $U_{p a} v s W$ dependence is an evidence of switching the mechanism of sound photogeneration. Visual observation of traces of failure indicates that the optical damage mechanism (the most probable is the laser ablation one) is predominant in this region. This is the superlinear dependence of $U_{p a}$ on $W$ that is predicted by a theory for mechanism of sound photogeneration associated with damage of material's structure [7]. It is worthwile to emphasize here that beginning of break of the $U_{p a}(W)$ curve, which is evidence of early stage of switching-on the sound photogeneration mechanism associated with damage of material, corresponds to the value of $W=2 \mathrm{MW} / \mathrm{mm}^{2}$ which is essentially lower than the value of $W=6-8 \mathrm{MW} / \mathrm{mm}^{2}$ of the visually observable damage. This is an immediate indication for higher sensitivity of the PA technique when applied to determination of a failure threshold.

Visually observable traces of damage of the sapphire for the III section are different from traces of the section II by presence of melting regions around the craters sizes of which are determined by the focused spot. From these data it may be concluded that in this region the melting process induced by the power laser irradiation is a main mechanism of the sound photogeneration and damage of a material. It shoud be noted that after a definite power density is attained, the plasma torch is occured. We suppose to investigate this aspect of the light-material interaction in our subsequent work.

\subsubsection{Modification of porous silicon lumines- cence properties}

Some works $[8,9]$ have demonstrated broad possibilities for employment the UV radiation also for enhancing the photosensitivity and changing parameters of luminescent emission of various materials. Significant results have been achieved in this direction when using the radiation of excimer lasers. Taking into account all the set forth above it seemed to be interesting to study the influence of $\mathrm{XeCl}$ *-laser irradiation of por-Si samples on their luminescent properties, in particular, on the shape of $I_{\text {lum }}(T)$ curve.

Por-Si samples obtained by applying the anodic selective etching to silicon plates oriented relatively to (100) crystallographic axis have been investigated. The etching had been carried out in an etchant consisting of $50 \%$
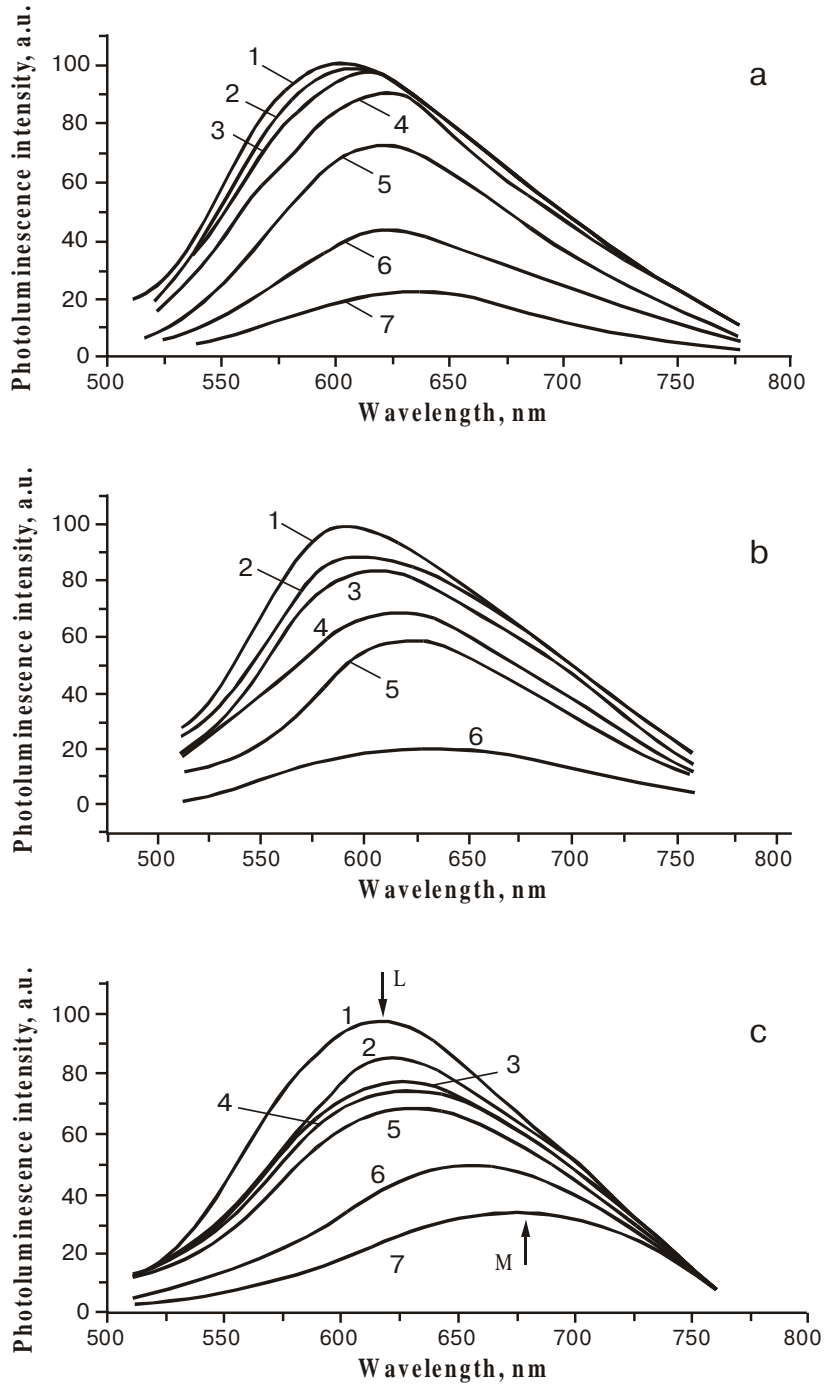

Fig. 7. Fragments of photoluminescence spectra of the 1st type samples, obtained under various temperature values (1-7) and UV-radiation doze (a-c): 1 - 5, 2-70, 3 - 100, 4 - 150, 5 - 200, $6-250,7-300 \mathrm{~K}$; a) non-irradiated sample, b) after 50 pulses of UV-radiation, c) after 150 pulses of UV-radiation.

of ethanol and 50\% of hydrofluoric acid. Details of formation of the por-Si layers are described in [10]. That is why here we only note that porosity of the samples, determined by the gravimetric method, constitutes $65 \%$ whereas the porous layers thickness equals to about $25 \mu \mathrm{m}$. Depending on etching conditions, all the samples investigated may be optionally broken into two groups differing by location of the main light emission band. These groups are: i) group 1 having the emission band maximum at $610 \mathrm{~nm}$ and ii) group 2 having the maximum at $720 \mathrm{~nm}$. Photoluminescence (PL) spectra have been measured by means of the diffraction spectrometer of «SDL$1 »$ type having a spectral slot width that did not exceed $0.1 \mathrm{~nm}$.

Irradiation of samples was performed by employing of designed $\mathrm{XeCl}^{*}$ eximer laser complex. When being 


\section{S.M.Baschenko, I.V.Blons'kiy: Science and technology excimer laser complex and...}
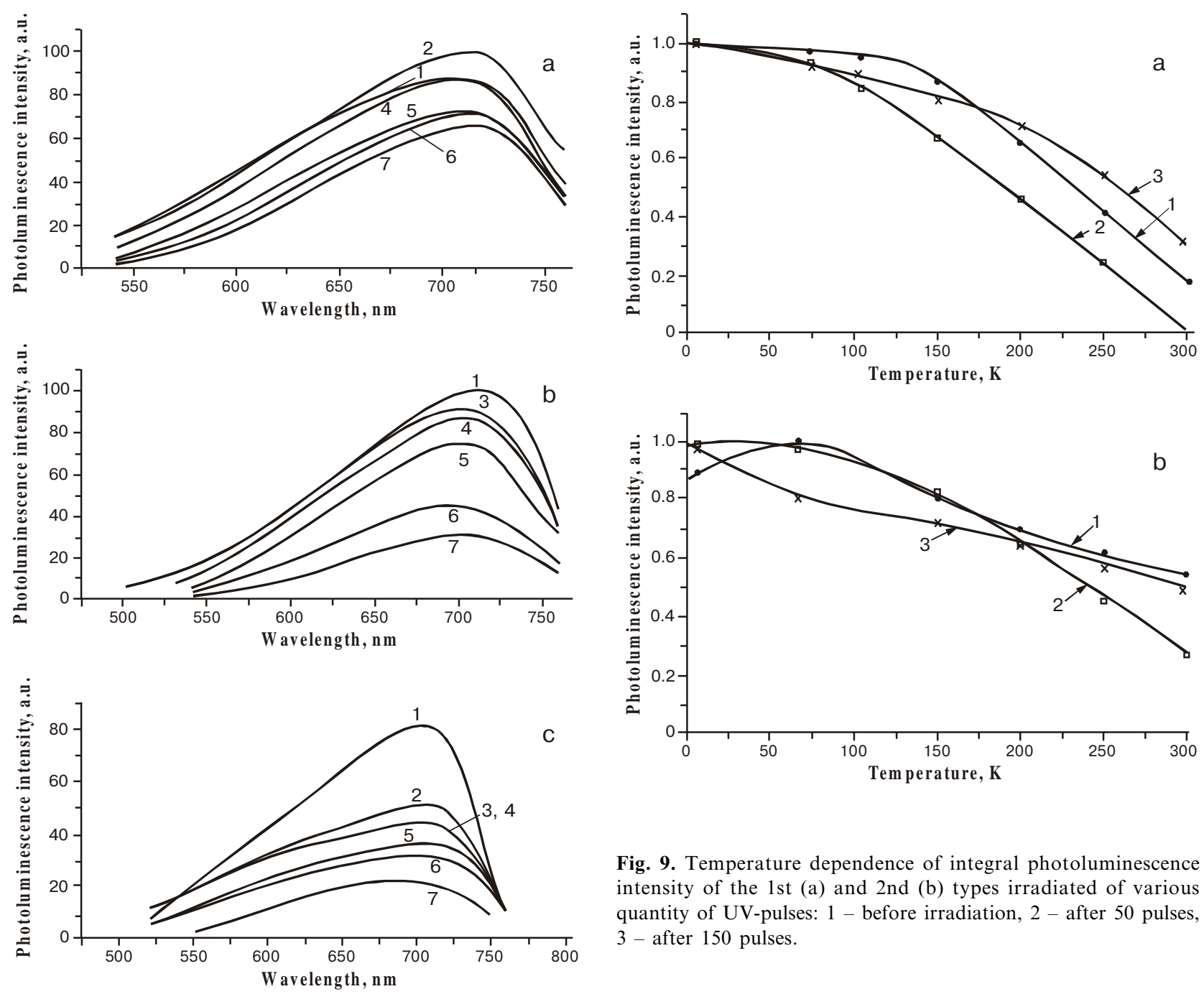

Fig. 9. Temperature dependence of integral photoluminescence intensity of the 1st (a) and 2nd (b) types irradiated of various quantity of UV-pulses: 1 - before irradiation, 2 - after 50 pulses, 3 - after 150 pulses.

Fig. 8. Fragments of photoluminescence spectra of the 2nd type samples, obtained under various temperature values $(1,2,4-7)$ and UV-radiation doze (a-c): 1 - 5, 2 - 70, 3 - 100, 4-150, $5-$ 200, $6-250,7-300 \mathrm{~K}$; a) non-irradiated sample, b) after 50 pulses of UV-radiation, c) after 150 pulses of UV-radiation.

irradiated, the samples were put into a special sealed chamber, which could be filled with various gases. The number of irradiation pulses could be varied (up to 150 pulses that correspond to the destruction threshold of the samples) in the $\mathrm{O}_{2}$-enriched atmosphere.

Fig. 7 and 8 show fragments of the PL spectra in the orange-red region measured at various temperatures $T$ (1-7), various doses of pulsed irradiation (a-c) for the samples of 1 st and 2 nd types, respectively. Fig. 9 shows a temperature dependence of integral photoluminescence intensity of 1st (a) and 2nd (b) types irradiated of various quantity of UV-pulses: 1 - before irradiation, 2 - after 50 pulses, 3 - after 150 pulses.

A main attention is paid to the following results:

- non-monotonic shape of $I_{l u m}(T)$ dependence for the initial samples of type 1 and typical - monotonic shape of such a dependence for the type 2 samples;

- change of shape of the $I_{\text {lum }}(T)$ depending on the irradiation dose: from non-monotonic shape to monotonic one for the type 1 samples (Fig. 9-a) as well as influence of the irradiation dose on magnitudes of coefficients $\left(d I_{\text {lum }} / d T\right)$ (Fig. 9-b) for the both types of samples;

- pronounced deformation of the band's curve shape as a function of temperature as a result of faster decay of its short-wave wing (this effect inherent to the type 1 samples only and becomes more pronounced under higher irradiation doses, Fig. 7 (a to c)).

As a result, spectral position of the maximum and shape of the light emission curve for the type 1 samples at room temperature (Fig. 7-c, spectrum 7) becomes identical to that for the type 2 samples spectra (Fig. 8, spectrum 7).

Therefore, the experimental results adduced in Fig. 7 to 9 testify that by irradiating the por-Si samples with nsec-pulses of the $\mathrm{XeCl}^{*}$-eximer laser it is possible to influence on the fundamental $I_{\text {lum }}(T)$ dependence as well as to modify PL spectra of the type 1 samples to be similar to those of the type 2 samples («7》) spectra in Fig. 7 and 9). The cause of this is that for the type 1 samples, in contrast with the type 2 samples, one can clearly distin- 
guish between two components ( $\mathrm{L}$ and $\mathrm{M}$ bands) within the whole emission range; each of these differently reacts upon both the temperature variation and the irradiation dose. In particular, UV-irradiation dose increasing for the type 1 samples reduces the temperature decay time of the L band (see Fig. 7 a-c). Such behavior may be associated with two reasons: i) lowering the energy barrier for transitions of carriers participating in the recombination emission from «radiative» to «non-radiative» state; ii) reduction of $\tau_{\mathrm{NR}}$ time of the non-radiative transitions. It should be noted that when changing energy parameters of the centers responsible for emergence of the L emission band, one waits the position of its maximum also has to be shifted, but really such a shift is not observed. This result forces us to put into basis of the model, explaining the experimental results of Fig. 7 to 9, the fact of UV irradiation influence on kinetic processes determining efficiency of the luminescent emission. Such an approach is consonant with the model proposed by the authors of [11] to explain the nature of non-monotonic feature of the $I_{l u m}(T)$ dependence which, as now it can be realized, is inherent to $\mathrm{M}$ component of the emission band.

At the same time it is known that the por-Si surface is passivated due to formation of the $\mathrm{SiOH}, \mathrm{SiH}$, and $\mathrm{SiO}_{x}$ compounds. Oxygen-containing Si compounds also promote the bright luminescence of por-Si. As to the hard radiation, it is accompanied, as a rule, first of all, by breaking the weak $\mathrm{Si}-\mathrm{H}$ bounds [12]. New complexes that are formed with participation of released hydrogen as well as interaction of silicon surface and atmosphere, can essentially influence onto kinetics of recombination of the photoexcited charge carriers. It is the base from which one should explain the UV-activation of the temperature decay rate of the $\mathrm{L}$ band (Fig. 7). In-depth description of the dependence $v s$ doze for the orange-red emission band decay of por-Si along with results of investigations of IR reflection spectra will be given in a separate work.

\section{Conclusion}

Preliminary testing and application of the complex have demonstrated its best performences, reliability, efficiency and suitability for wide tasks range as promising of suggested original scheme. Applicability of the complex for various tasks (processing of super-hard non-metallic materials in non-contact, non-thermal regime, distance atmosphere monitoring, porous silicon treatment and modification of its properties) is demonstrated.

For the first time it has been demonstrated a possibility to influence by UV-irradiation on the shape of $I_{\text {lum }}(T)$ dependence in the region of orange-red luminescence band for the por-Si samples. In the type 1 samples two spectral components have been elucidated, each differently reacting on the UV-irradiation phase and on temperature change. It is concluded that different shapes of the $I_{l u m}(T)$ dependence previously observed on various samples by different groups of investigators is determined by different active contributions into resulting shape of the L, M luminescence bands and, possibly, other emission bands. Nature of the UV irradiation influence on the $I_{l u m}(T)$ dependencies is analysed.

Together with a main results some adding ones was demonstrated. So, ability of the PA technique to determine the failure threshold of processed sapphire plates was demonstrated. The dependence of PA response amplitude $U_{p a}$ on irradiation power density $\mathrm{W}$ is determined for the range of $0.6-300 \mathrm{MW} / \mathrm{mm}^{2}$. Three characteristic sections of the $U_{p a}(W)$ dependence are found, in which different mechanisms of sound photogeneration predominate: thermoelastic mechanism (I), mechanism involving ablation (II), and mechanism involving melting (III). Advantage of employment of the eximer $\mathrm{XeCl}^{*}$ laser for a high-precious processing of the sapphire and other hard materials is illustrated.

Together with the above some complexities was revealed, too.

It shows up when passing to «oscillator-amplifier» scheme of SRS-conversion for higher SRS Stokes and antiStokes component obtaining. Main complexity lies in need of very precise and rigid mutual stability of optical component position, because the SRS-amplification (and generation) is takes place in very small volume, and any vibrations or shifts over comparable distance sharply decrease or even exclude SRS-amplification. It showing up to us that solving of rigid fixing problem lies on the way of mono-block construction of SRS-converter.

\section{References}

1. V.Ischenko, N.Karlov et al., Measure of Ittherbiy photoionization cross-section from excited state ${ }^{3} \mathrm{P}_{1} / /$ Letters to the JTP, 20, p.1044-1048, (1977)

2. E. Hinckley, et al. // Laser Monitoring of the Atmosphere, Springer-Verlag, . (1976)

3. R. Measures, Laser Remote Sensing, John Willey\&Sons, New York (1984)

4. Herzberg G., Molecular Spectra and Molecular Structure III. Electronic spectra and electronic structure of polyatomic molecules. Van Nostrand, Princeton, N.J.(1967).

5. Vassy E., Property of Ozone // Ann. De Phys., 8 (11), 679, (1937).

6. S.M.Baschenko, I.V.Blonskij, V.M.Puzikov et al. Photoacoustic techniques for determination of threshold of failure during laser processing of substrate materials for integrated electronics. // SPIE v.3359, pp 519-522, (1997)

7. V.E.Gusev, A.A.Karabutov, // Laser Optoelectronics, p.234, «Nauka», Moscow, (1991).

8. N.Noguchi, I.Suemune. Luminiscent porous silicon synthesized by vizible light irradiation.// Appl.Phys.Lett. 62 (12), pp.1429-1431 (1993).

9. A.Anedda, G.Bongiovanni, M.Cannas at al. A 1,9 eV photoluminiscence induced by $4 \mathrm{eV}$ photons in high-purity wet synthetic silica.// J.Appl.Phys. 74, p.6993 (1993).

10. R.L.Smith, S.D.Collins. Porous Silicon formation mechanism. // J.Appl. Phys. 71(8), pp.R1-R22 (1992)

11. M.Rosenbauer, M.Stutzmann, H.Fuchs at al. Temperature dependence of luminiscence in porous silicon and related materials.// J.of luminiscence. 57, p.153 (1993).

12. E.V. Astrova, R.F. Vitman, Yu.V. Rud' et al. Influence of the $\gamma$-irradiation on porous silicon properties // PhTP, 30(3), p.507 (1996). 